\title{
Das ,framing“ der sechsmonatigen Karenzregel in der Lebertransplantation. Ein Beispiel für sprachlich vermittelte Deutungsmuster zur Eingrenzung des Indikationsgebietes
}

\author{
Nadia Primc $\mathbb{D}$
}

Eingegangen: 16. Januar 2020 / Angenommen: 27. April 2020 / Online publiziert: 26. Mai 2020 (C) Der/die Autor(en) 2020

Zusammenfassung Die deutschen Richtlinien zur Lebertransplantation sehen vor, dass Patient*innen mit alkoholbedingten Lebererkrankungen in der Regel eine sechsmonatige Karenz nachweisen müssen, bevor sie auf die Warteliste für eine Lebertransplantation aufgenommen werden können. Die international weit verbreitete Karenzregel wurde von Beginn an sehr kritisch diskutiert, da hiermit Patient*innen eine wirksame und potentiell lebensrettende Therapie zumindest vorübergehend vorenthalten wird. Sie kommt in der Praxis einer (vorübergehenden) Eingrenzung der Indikation zur Lebertransplantation gleich. Aus der medizinischen Fachliteratur lassen sich vier Interpretationsrahmen rekonstruieren, die mittels Herausstellung bestimmter Aspekte eine jeweils unterschiedliche Wahrnehmung und Konzeptualisierung der sechsmonatigen Karenzregel begründen. Diese sprachlich vermittelten Deutungsmuster werden von Akteur*innen zur Eingrenzung der Indikation zur Lebertransplantation genutzt. Innerhalb dieser Interpretationsrahmen wird die sechsmonatige Karenzzeit jeweils 1) als diagnostisches Werkzeug zur Bestimmung der Regenerationsfähigkeit der Leber, 2) als prognostisches Werkzeug zur Bestimmung der Erfolgsaussichten einer Transplantation, bzw. 3) zur Bestimmung des Trinkverhaltens nach Transplantation dargestellt. In einem weiteren Rahmen wird die Karenzregel 4) als Ausdruck einer Verpflichtung zur Vermeidung von gesundheitsschädlichem Verhalten interpretiert. Aus diesen vier Interpretationsrahmen ergeben sich unterschiedliche ethische Fragestellungen, die eine systematische ethische Beurteilung der Karenzregel ermöglichen. In den ersten drei Interpretationsrahmen wird wiederum selbst die Frage nach der medizinischen Indiziertheit der Karenzregel aufgeworfen.

Schlüsselwörter Lebertransplantation · Alkoholkarenz · Organallokation · Medizinische Indikation · Framing 


\title{
The framing of the six-month abstinence rule in liver transplantation. An example of linguistically mediated patterns of interpretation used to limit indication area
}

\begin{abstract}
Background The German guidelines for liver transplantation stipulate that every patient with alcohol-related liver disease needs to prove evidence of a 6-month abstinence period before they can be admitted to the waiting list for liver transplantation. This internationally widespread abstinence rule has been criticised as it prevents patients at least temporarily from receiving an effective and potentially lifesaving therapy. This poses the question of how this abstinence rule is depicted and justified by transplantation professionals.

Arguments In case of the 6-month abstinence rule, guidelines are used as a tool for rationing health care. Four frames of interpretation can be reconstructed from the ethical and medical literature. Each linguistically mediated frame offers a different representation of the 6-month abstinence rule. According to these different framings, the 6-month waiting period is depicted (1) as a diagnostic tool to determine the regenerative capacity of the liver, (2) as a prognostic tool to determine the prospects of success of a transplantation, and (3) to determine the drinking behaviour after transplantation. In a further context, the abstinence rule (4) is interpreted as an expression of an obligation to avoid behaviour harmful to health.

Conclusion These four frames provide linguistically mediated patterns of interpretation that are used by different actors to limit the indication for liver transplantation. They give rise to different ethical questions that allow a systematic ethical assessment of the 6-month abstinence rule.
\end{abstract}

Keywords Liver transplantation - Abstinence rule $\cdot$ Organ allocation - Medical indication · Framing

\section{Einleitung}

International weit verbreitet ist die sogenannte sechsmonatige Karenzregel, die Patient*innen mit alkoholbedingten Lebererkrankungen häufig erfüllen müssen, wenn sie Zugang zur Warteliste für eine Lebertransplantation erhalten möchten. Im Gegensatz zu anderen Ländern, in denen die Organvergabe und Entscheidung über die Aufnahme auf die Warteliste regionalen und zentrumsspezifischen Unterschieden unterworfen ist, kommt den Richtlinien der Bundesärztekammer (BÄK) bundesweite und strikte Geltung zu.

Die Forderung einer mehrmonatigen Karenz wurde auf internationaler Ebene von Beginn an kontrovers diskutiert, da hierdurch Patient*innen eine an sich wirksame und potentiell lebensrettende Ressource zumindest temporär vorenthalten wird. Es handelt sich hierbei um eine begründungsbedürftige Eingrenzung des Indikationsgebiets. Die Richtlinien der BÄK werden hier als Instrument der Leistungsbegrenzung für eine an sich wirksame Therapie genutzt. 
Die anhaltende Diskussion rund um die Zulässigkeit der sechsmonatigen Karenzregel ist zum Teil auch auf eine unzureichende Differenzierung der unterschiedlichen Interpretationsrahmen zurückzuführen, in welche die Karenzregel von den Akteur*innen jeweils gestellt wird. Im Folgenden werden diese sprachlich konstituierten Interpretationsrahmen, die jeweils als Instrument zur Eingrenzung des Indikationsgebiets genutzt werden, an Hand der medizinischen Literatur rekonstruiert und einige der sich hieran anschließenden ethischen Fragestellungen diskutiert.

\section{Leit- und Richtlinien als Instrumente der Leistungsbegrenzung und Einschränkung des Indikationsgebietes}

Der Begriff der medizinischen Indikation (bzw. der Prozess der Indikationsstellung) wird in der medizinethischen Literatur vornehmlich in normativer Hinsicht diskutiert, d.h. es geht zumeist um die Frage, wie der Begriff der medizinischen Indikation aus ethischer Sicht definiert und in der medizinischen Praxis verwendet werden soll. Als Minimalkonsens lässt sich feststellen, dass der Begriff der medizinischen Indikation unter anderem das Urteil enthält, dass eine bestimmte medizinische Maßnahme mit hinreichend großer Wahrscheinlichkeit dazu geeignet ist, ein bestimmtes Therapieziel zu erreichen, wie etwa die Lebensqualität zu verbessern oder die Lebenserwartung zu erhöhen (Maio 2015, S. 76f.; Marckmann 2015b, S. 118 f.; Neitzke 2015, S. 84 f.; Wiesing 2017, S. 54 f.). Entsprechend wird es als kritikwürdig angesehen, wenn dieses Urteil durch Interessen beeinflusst wird, die jenseits der Orientierung am Wohl oder Willen der Patient*innen liegen, wie etwa ökonomische oder sonstige Eigeninteressen einer Institution oder der behandelnden Ärzt*innen (Wiesing 2017, S. 129 ff.). Dies wäre etwa dann der Fall, wenn eine an sich geeignete Maßnahme allein deswegen gegenüber Patient*innen als nicht indiziert oder gar kontraindiziert ausgewiesen wird, weil die Vergütung von den Leistungserbringern als wenig lukrativ wahrgenommen wird.

Eine eher rekonstruierende Herangehensweise fokussiert hingegen darauf, wie in der Praxis die Indiziertheit einer medizinischen Intervention zwischen Akteur*innen verhandelt wird. Wie bereits angedeutet, besteht die Gefahr, dass in der Praxis das Indikationsgebiet eingeschränkt wird, obwohl eine medizinische Intervention an sich geeignet wäre, die Lebensqualität einer bestimmten Patient*innengruppe zu verbessern, bzw. ihre Lebenserwartung deutlich zu erhöhen. Unter einem Indikationsgebiet ist hierbei die Angabe der gesundheitlichen ,Zustände“, d. h. der medizinischen Diagnosen zu verstehen, bei welchen eine Intervention zur Erreichung eines bestimmten therapeutischen, diagnostischen oder präventiven Zieles als angezeigt anzusehen ist (Wiesing 2017, S. 72 f.). Die Bestimmung und Einschränkung einzelner Indikationsgebiete ist vornehmlich Aufgabe von Fachgesellschaften und Expertengremien. Sie erfolgt zumeist in Form von allgemeinen Expertenstandards, Leit- oder auch Richtlinien und basiert auf den zu einem gegebenen Zeitpunkt verfügbaren Studienergebnissen, d.h. dem gegenwärtigen Stand der medizinischen Forschung (Wiesing 2017, S. $70 \mathrm{ff}$.). Die Verhandlung der Reichweite und Grenzen des Indikationsgebietes lässt sich in diesen Fällen methodisch anhand der inhaltlichen Analyse der entspre- 
chenden Leit- und Richtlinien sowie der sich hierauf beziehenden Fachpublikationen oder auch Patient*inneninformationen rekonstruieren.

\section{Die sechsmonatige Karenzzeit bei alkoholbedingten Lebererkrankungen als (vorübergehende) Einschränkung des Indikationsgebiets}

In den aktuellen Richtlinien der BÄK (2019) ist vorgesehen, dass Patient*innen mit alkoholinduzierter Zirrhose eine sechsmonatige Abstinenz nachweisen müssen, um Zugang zur Warteliste für Lebertransplantationen zu erhalten. Eine Änderung der Richtlinien im Jahr 2015, die vornehmlich auf die Kritik daran zurückzuführen ist, dass eine Reihe von Patient*innen, insbesondere jene mit einer akuten alkoholischen Hepatitis, die sechsmonatige Karenzzeit häufig nicht überleben (Al-Saeedi et al. 2018; Mathurin et al. 2011; Wu et al. 2018), ermöglicht es den Transplantationszentren seitdem, für Patient*innen mit einer akut dekompensierten Lebererkrankung eine Ausnahme von der Karenzregel zu beantragen (Bundesärztekammer 2019).

In der Praxis kommt die Karenzregel einer rechtfertigungswürdigen Einschränkung der Indikation zur Lebertransplantation gleich. So wird in den Richtlinien der BÄK die Leberzirrhose explizit als eine der häufigsten Indikationsgruppen für eine Lebertransplantation aufgeführt. Zugleich ist davon die Rede, dass Patient*innen in die Warteliste aufgenommen werden können, ,wenn die Überlebenswahrscheinlichkeit und/oder die Lebensqualität mit Transplantation größer ist als ohne“ (Bundesärztekammer 2019) - eine Definition, die im Wesentlichen dem oben beschriebenen Minimalkonsens hinsichtlich des Begriffs der medizinischen Indikation entspricht. Dass Letzteres auch auf viele Patient*innen ohne entsprechende Karenzzeit zutrifft, wird mittlerweile von einer ganzen Reihe von Studien belegt (vgl. Al-Saeedi et al. 2018; Lee et al. 2018; Obed et al. 2018; Wu et al. 2018). Die Forderung eines derartigen Karenznachweises wird nicht erst seit der Studie von Mathurin et al. (2011) zunehmend in Frage gestellt, die international den Anstoß für eine ganze Reihe von weiteren Studien zur frühzeitigen Lebertransplantation ohne sechsmonatige Karenzzeit gegeben hat (vgl. Im et al. 2018; Wu et al. 2018). Sie wurde vielmehr von Beginn an sehr kontrovers diskutiert (vgl. Cohen et al. 1991; Glannon 2009; Moss und Siegler 1991).

Dass die fehlende Alkoholkarenz, wenn auch nicht explizit sprachlich so benannt, in den Richtlinien der BÄK in inhaltlicher Nähe zu einer Kontraindikation oder Einschränkung des Indikationsgebietes zu verstehen ist, wird dadurch bestärkt, dass Patient*innen mit alkoholinduzierten Lebererkrankungen, die nach der Aufnahme auf die Warteliste Alkohol konsumieren, als ,nicht transplantabel“ (NT) an die Vermittlungsstelle gemeldet werden müssen. Die NT-Listung ist in den Richtlinien explizit für vorübergehende Kontraindikationen vorgesehen (Bundesärztekammer 2019). Dass die Karenzregel in der praktischen Folge für die betroffenen Patient*innen einer Kontraindikation zur Lebertransplantation gleich kommt, zeigt sich unter anderem daran, dass auch in der neueren medizinischen Fachliteratur das Nichteinhalten der Karenz ausdrücklich als Kontraindikation bei Lebertransplantation wahrgenommen wird (vgl. Al-Saeedi et al. 2018; Im et al. 2018; Mathurin et al. 2011). 
Da die strikte Alkoholkarenzforderung Patient*innen von einer potentiell lebenserhaltenden Therapie ausschließt und in der Praxis einer (vorübergehenden) Einschränkung des Indikationsgebietes gleichkommt, stellt sich die Frage, wie sich diese Regelung medizinisch und ethisch rechtfertigen lässt. Aus den Richtlinien und der Fachliteratur der letzten drei Jahrzehnte seit Einführung der sechsmonatigen Karenzregel in den Fachdiskurs - eine explizite Festlegung auf sechs Monate wird bereits von Bird et al. (1990) gefordert - lassen sich vier Interpretationsrahmen (,frames“ oder ,framings“) rekonstruieren, in welche die sechsmonatige Karenzregel jeweils gestellt wird. Diese sprachlich vermittelten Interpretationsrahmen stellen Deutungsmuster zur Verfügung, aus denen sich mittels Herausstellung bestimmter Aspekte eine unterschiedliche Wahrnehmung und Konzeptualisierung der sechsmonatigen Karenzregel ergeben (zum Begriff des „framing“ siehe Entman 1993). An diese schließen sich wiederum unterschiedliche Begründungsstrategien zur Rechtfertigung der geforderten sechsmonatigen Karenz an. Die Orientierung an den vier Interpretationsmodellen ermöglicht demnach eine Systematisierung der ethischen Debatten.

Die Recherche der medizinischen Fachliteratur erfolgte mittels PubMed unter Verwendung der Titel- und/oder Abstract-Stichwörter ,,alcohol“ und ,liver transplant“. Die Suche wurde auf klinische Studien, Reviews und Zeitschriftenartikel beschränkt und ergab 335 Treffer. Nach Durchsicht der Abstracts wurde für 67 Beiträge nach dem Volltext gesucht sowie aus den Literaturverzeichnissen dieser Beiträge weitere Literaturhinweise entnommen und recherchiert. Nach Durchsicht der Beiträge hinsichtlich ihrer Relevanz für die Untersuchung des ,framing“ der sechsmonatigen Karenzzeit wurden insgesamt 49 Beiträge in die inhaltliche Analyse eingeschlossen. Für die ethische Fachliteratur wurden insgesamt 11 nationale und internationale Fachzeitschriften zur Bioethik sowie zur Ethik der Medizin und Gesundheitsversorgung mittels der Stichwörter ,alcohol“ und „liver transplantation“ (bzw. ,Alkohol“ und „Lebertransplantation“) durchsucht und aus den entsprechenden Beiträgen weiterführende Literaturverweise entnommen. Insgesamt wurden nach der inhaltlichen Durchsicht 56 Beiträge in die weitere ethische Untersuchung einbezogen.

\section{Vier unterschiedliche Formen des „framing“ der sechsmonatigen Karenzzeit}

Framing essentially involves selection and salience. To frame is to select some aspects of a perceived reality and make them more salient in a communicating text, in such a way as to promote a particular problem definition, causal interpretation, moral evaluation, and/or treatment recommendation for the item described (Entman 1993, S. 52).

Beim „framing“ handelt es sich um einen vornehmlich aus der Wissenssoziologie und kognitiven Psychologie stammenden Fachbegriff, der seit den 1970er Jahren zunehmend Eingang in andere geistes-, kultur- und sprachwissenschaftliche Fachgebiete gefunden hat. Eine wesentliche Gemeinsamkeit der fachspezifischen Verwendungsweisen des Begriffs des ,framing“ lässt sich mit Entman darin ver- 
orten, dass durch sprachlich vermittelte Interpretationsrahmen bestimmte Aspekte eines Phänomens in den Vordergrund, andere hingegen in den Hintergrund gerückt werden. Durch die reflektierte Wahl eines Interpretationsrahmens lässt sich demnach die Wahrnehmung eines bestimmten Phänomens, in diesem Fall der Karenzregel, wesentlich beeinflussen.

Neben einer epistemologischen enthält ein ,framing“ zugleich auch immer eine evaluative oder gar ethisch-normative Komponente. Die im Folgenden vorgestellten vier unterschiedlichen „framings“ der sechsmonatigen Karenzzeit unterscheiden sich dennoch hinsichtlich der Zentralität der ethisch-normativen Gesichtspunkte. Während die ersten drei „frames“ stärker medizinische Aspekte der sechsmonatigen Karenzzeit in den Vordergrund stellen, zielt der vierte Interpretationsrahmen vornehmlich auf die Verantwortung der Patient*innen auf der Warteliste ab.

\section{Die sechsmonatige Karenzzeit als diagnostisches Werkzeug zur Bestimmung der Regenerationsfähigkeit der Leber}

Der von Seiten der Transplantationsmedizin am stärksten in den Vordergrund gerückte Interpretationsrahmen hebt die Wirksamkeit und den Nutzen der sechsmonatigen Karenzzeit zur Bestimmung des natürlichen Regenerationspotentials der Leber hervor (Bundesärztekammer 2019). In einigen Fällen können durch eine strikte Karenz die Funktion der Leber und der Gesundheitszustand der Patient*in verbessert werden. Die Entfernung des zentralen Hepatotoxins schafft eine günstigere Ausgangssituation für die geplante Lebertransplantation oder kann dazu führen, dass Letztere verschoben oder gar nicht mehr notwendig wird (Mellinger und Volk 2018, S. 174; Testino et al. 2014, S. 14.647 f.; Bramstedt und Jabbour 2006, S. 263; Donckier et al. 2013, S. 867). Die sechsmonatige Karenzzeit wird hier als diagnostisches Werkzeug zur Unterscheidung einer rückbildungs- von einer nicht-rückbildungsfähigen Leberschädigung beschrieben und soll es ermöglichen, den Grad der Regenerationsfähigkeit näher zu bestimmen. Hierdurch soll sichergestellt werden, dass alle konservativen Therapiemöglichkeiten ausgeschöpft und die Lebertransplantation als risikobehaftete und stark invasive Maßnahme tatsächlich die letzte verbleibende Therapiemöglichkeit darstellt.

\section{Die sechsmonatige Karenzzeit als prognostisches Werkzeug zur Bestimmung der Erfolgsaussichten einer Transplantation}

Ein ebenfalls weit verbreiteter Interpretationsrahmen verortet die medizinische Berechtigung und den Nutzen der sechsmonatigen Karenzregel darin, dass hierdurch der mittel- und langfristige Transplantationserfolg sichergestellt werden soll, im Sinne des Patient*innenüberlebens und/oder der Transplantatsfunktion (vgl. Al-Saeedi et al. 2018; Batra und Wiesing 2018, S. 8; Im et al. 2018; Lee et al. 2018; Marroni et al. 2018; Obed et al. 2018). Gemäß diesem Deutungsmuster stellt die Karenzzeit ein prognostisches Werkzeug zur Bestimmung der Erfolgsaussichten einer Transplantation dar. 


\section{Die sechsmonatige Karenzzeit als prognostisches Werkzeug zur Bestimmung des Trinkverhaltens nach Transplantation}

Eng verbunden mit dem vorherigen Interpretationsrahmen ist die Überzeugung, dass die sechsmonatige Karenzzeit die Wahrscheinlichkeit eines Rückfalls nach Transplantation minimiert, was auch Auswirkungen auf den Transplantationserfolg haben kann (vgl. Al-Saeedi et al. 2018; Im et al. 2018; Lee et al. 2018, S. 361 ff.; Marroni et al. 2018; Obed et al. 2018; Testino et al. 2014). Auch hier wird die Karenz als technologisch wenig aufwändiges Prognoseinstrument, nämlich zur Vorhersage des postoperativen Trinkverhaltens beschrieben.

\section{Die sechsmonatige Karenzzeit und die Verantwortung zur Vermeidung von gesundheitsschädlichem Verhalten}

Ein stärker moralisierendes Deutungsmuster liegt all jenen Äußerungen zugrunde, die eine sechsmonatige Karenzzeit nicht nur aus rein medizinischen Gründen fordern, sondern hierin auch die ethische Verantwortung der Patient*innen sehen, den gesundheitsschädlichen Alkoholkonsum einzustellen, wenn sie Zugang zu der äußerst knappen Ressource der Spenderorgane erhalten wollen (prospektive Verantwortung). Dieser Interpretationsrahmen unterscheidet sich von dem vorherigen dadurch, dass hier nicht die Sicherheit einer Prognose verhandelt wird, sondern die Verantwortlichkeit und der damit einhergehende ethische Anspruch einer Patient*in auf die knappe Ressource der Spenderorgane. Nur wer tatsächlich bemüht ist, sein schädigendes Trinkverhalten einzustellen, sollte auch Anspruch auf ein Spenderorgan haben. Hiermit eng verbunden ist die Vorstellung, dass Patient*innen mit alkoholbedingten Lebererkrankungen zumindest teilweise für ihre lebensbedrohliche Situation verantwortlich sind (retrospektive Verantwortung), d.h. eine gewisse Mitschuld tragen, und daher bei der Vergabe der Spenderorgane zu einem gewissen Grade nachteilig berücksichtigt werden sollten (Donckier et al. 2013; Cohen et al. 1991; Glannon 2009; Ho 2008; Marroni et al. 2018, S. 2787; Moss und Siegler 1991; Solga et al. 2018; Veatch und Ross 2015, S. 310 ff.; Zambrano 2016).

Ebenfalls unter diesen Interpretationsrahmen zu subsumieren ist die Befürchtung, dass die Aufhebung der Karenzforderung zu einer geringeren Spendebereitschaft in der Bevölkerung führen könnte (Wu et al. 2018). Denn hiermit wird unterstellt, dass Patient*innen mit alkoholbedingten Lebererkrankungen seitens der Öffentlichkeit kein gleichwertiger Anspruch auf die knappe Ressource der Spenderlebern oder ein gewisser Grad an Eigenverantwortung für ihre lebensbedrohliche Situation zugesprochen wird.

\section{Die ethische Bewertung der vier Interpretationsrahmen}

Die in den einzelnen „frames“ enthaltenen unterschiedlichen Darstellungen und Wahrnehmungen der Karenzregel geben Anlass zu verschiedenen ethischen Fragestellungen, die im vorliegenden Beitrag nicht alle im Detail diskutiert werden können. Da bei den ersten drei Interpretationsrahmen die Frage der medizinischen 
Indiziertheit wiederum eine besondere Rolle spielt, wird im Folgenden vornehmlich auf diese eingegangen. Die sich an das vierte ,framing“ anschließenden Fragen der retrospektiven und prospektiven Verantwortung führen über den in dem vorliegenden Themenheft behandelten Themenbereich der medizinischen Indikation hinaus und können daher nicht näher erörtert werden. $\mathrm{Zu}$ nennen sind hier insbesondere die Frage, inwiefern retrospektive und/oder prospektive Verantwortung als ein ethisch zulässiges Kriterium für die Verteilung von Spenderlebern anzusehen ist und inwiefern Suchterkrankungen dem Bereich der retrospektiven oder auch prospektiven Verantwortung zugerechnet werden können (Cohen et al. 1991; Glannon 2009; Ho 2008; Obed et al. 2018; Thornton 2009; Veatch und Ross 2015, S. $310 \mathrm{ff.;}$ Zambrano 2016). Die Fokussierung auf eine ethische Bewertung der drei ersten Interpretationsrahmen ist auch insofern gerechtfertigt, da die ethische Fachliteratur sich in der Bewertung der Karenzregel bisher vornehmlich auf die Diskussion des vierten „framings“ und die Frage der retrospektiven und prospektiven Verantwortung als Allokationskriterium konzentriert hat. Zu den ersten drei „framings“ finden sich größtenteils nur kürzere ethische Stellungnahmen, Kommentare und Editorials, so dass hier eine wichtige Lücke in der ethischen Diskussion zumindest ansatzweise geschlossen werden kann.

\section{Die medizinische Indiziertheit der Karenzregel}

Die sechsmonatige Karenzzeit wird im Rahmen der ersten drei „frames“ als medizinische Maßnahme mit diagnostischer bzw. prognostischer Funktion gedeutet. Hier stellt sich somit selbst wiederum die Frage nach der Indiziertheit, d. h. inwiefern die Sechsmonatsregel für das Indikationsgebiet der alkoholbedingten Lebererkrankungen tatsächlich ein geeignetes Diagnose- bzw. Prognoseinstrument darstellt. Während in besonderen Fällen als letztes Mittel zur Lebensrettung auch der Einsatz von unerprobten Interventionen unter Umständen ethisch gerechtfertigt werden kann, ist von medizinischen Maßnahmen im Allgemeinen zu fordern, dass sie gemäß dem gegenwärtigen Stand der medizinischen Wissenschaft als indiziert anzusehen sind. Der Einsatz von medizinischen Interventionen setzt voraus, dass aufgrund der vorliegenden klinischen Studienergebnisse mit hinreichend großer Sicherheit erwartet werden kann, ein bestimmtes therapeutisches, diagnostisches oder präventives Ziel $\mathrm{zu}$ erreichen.

\section{Die Karenzzeit als diagnostisches Werkzeug zur Bestimmung der Regenerationsfähigkeit der Leber}

Der Einsatz der Karenzregel als Mittel zur Bestimmung der Regenerationsfähigkeit der Leber wird unterschiedlich ethisch gerechtfertigt. Die Gründe lassen sich in patienten- und allokationsorientierte Gründe unterteilen (Marckmann 2015a). Patientenorientierte Gründe heben hervor, dass die allgemeine Verbesserung des Gesundheitszustandes oder sogar Vermeidung einer Lebertransplantation mit lebenslanger Immunsuppressionstherapie im Interesse der Patient*innen ist. Es ist jeweils sicherzustellen, dass es sich hierbei um die letzte zur Verfügung stehende Therapieform handelt (Bramstedt und Jabbour 2006, S. 263; Donckier et al. 2013, S. 867; Mellin- 
ger und Volk 2018, S. 174; Testino et al. 2014, S. 14.647f.). Allokationsorientierte Argumente betonen hingegen die positiven Effekte für das gesamte Kollektiv der Lebertransplantationskandidat*innen: Als Folge stehen insgesamt mehr Spenderlebern zur Verfügung und Retransplantationen aufgrund eines schlechten Gesundheitszustandes können vermieden werden (Greif-Higer 2015; Marckmann 2015a; Veatch und Ross 2015, S. 307).

Kritisch ist gegen die patientenorientierte Rechtfertigung der Karenzregel anzumerken, dass nicht alle Patient*innen diese Karenzzeit überleben sowie die Frage der Regenerationsfähigkeit der Leber sich auf andere Weise besser überprüfen lässt als vermittels einer starren Karenzregel (Mellinger und Volk 2018, S. 174). So ist in diesem Kontext zum Beispiel zu prüfen, ob drei Monate nicht eine angemessenere Zeitspanne zur Feststellung der Regenerationsfähigkeit der Leber darstellen sowie präzisere klinische Kriterien hinzugezogen werden können (Mathurin et al. 2011; Testino et al. 2014, S. 14.647). Ein diagnostisches Verfahren ist nur dann als indiziert anzusehen, wenn es in einem bestimmten Fall dazu geeignet ist, Wissen zu generieren, das es den behandelnden Ärzt*innen ermöglicht, die therapeutischen Indikationen präziser zu stellen, d.h. zu bestimmen, welche therapeutischen Interventionen aus medizinischer Sicht das beste Risiko-Nutzen-Profil aufweisen (Wiesing 2017, S. 64). Die Vermeidung eines unnötigen und riskanten Eingriffes stellt ohne Zweifel aus Sicht des Patientenwohles ein hehres und wichtiges Ziel dar. Allerdings birgt der Einsatz der Sechsmonatsregel als diagnostisches Werkzeug zumindest in der gegenwärtigen in Deutschland angewendeten Form unverhältnismäßig große Risiken für einige Patient*innen. Nämlich dann, wenn diese trotz der in den Richtlinien der BÄK empfohlenen suchtmedizinischen Betreuung einen oder mehrere Rückfälle erleiden, d.h. Alkohol in unbestimmter Menge konsumieren, sodass die lebensrettende Transplantation für sie aufgrund der gegenwärtigen Karenzregel immer wieder in weite Ferne rückt. Die Einführung eines diagnostischen Tests mit einem derartigen Risikoprofil würde in jedem anderen Bereich der Medizin wohl als unverhältnismäBig und ethisch nicht gerechtfertigt abgelehnt werden.

Soll die Karenzzeit (im Sinne einer patientenorientierten ethischen Begründung) als diagnostisches Werkzeug zur Identifikation der bestmöglichen Therapie für die betroffenen Patient*innen dienen, wäre eine modifizierte Anwendung derselben in der Praxis gefordert. Eine derartige modifizierte Anwendung könnte so aussehen, dass Patient*innen nach entsprechender Aufklärung mittels suchtmedizinischer Betreuung dabei unterstützt werden, zur Bestimmung der Notwendigkeit einer Lebertransplantation und der Regenerationsfähigkeit ihrer Leber, jeden weiteren Alkoholkonsum abzustellen, ohne dass ein Rückfall per se immer wieder zum sechsmonatigen Ausschluss von der Warteliste führt (Veatch und Ross 2015, S. $315 \mathrm{f}$.). Auf dieser Grundlage ließe sich eine wahrhaft patientenorientierte Anwendung der Karenzforderung ausgestalten, die versucht, für die Patient*innen die bestmögliche Therapieform zu identifizieren, ohne all diejenigen Patient*innen auszuschließen, die die Einhaltung der strikten Karenzregel nicht in der vorgegebenen Zeit zu realisieren vermögen. Hieran lässt sich auch die von ethischer Seite geforderte „Umkehr der Beweislast" anschließen (Marckmann 2015a), sodass nicht mehr wie bisher in Deutschland von den behandelnden Ärzt*innen für jeden Einzelfall eine Ausnahme von der Karenzregel beantragt werden muss, sondern umgekehrt, vielmehr das 
Fehlen hinreichender Erfolgsaussichten im Einzelfall belegt werden muss, um den Patienten in begründeter Weise die Aufnahme auf die Warteliste zu verweigern.

Die angesprochene allokationsorientierte Begründung, welche die insgesamt gröBere Anzahl an Spenderorganen für die verbleibenden Patient*innen betont, ist auf der Grundlage allokationsethischer Überlegungen zu bewerten. Sie gründet auf einer bestimmten Auslegung des Kriteriums der Erfolgsaussicht, nämlich der Forderung, so viele Menschenleben wie möglich zu retten, und leitet damit zu dem zweiten und dritten Interpretationsrahmen, dem Gebrauch der Karenzregel als Werkzeug zur Bestimmung der Erfolgsaussichten und des Trinkverhaltens nach Transplantation über.

Die Karenzregel als prognostisches Werkzeug zur Bestimmung der Erfolgsaussichten und des Trinkverhaltens nach Transplantation

Angesichts der beiden „framings“, die die sechsmonatige Karenzzeit jeweils als prognostisches Werkzeug zur Bestimmung der Erfolgsaussichten der Transplantation oder des Trinkverhaltens nach Transplantation verstehen, stellt sich die Frage nach der Präzision des Prognoseinstrumentes. Beide Interpretationsrahmen hängen auch insofern eng miteinander zusammen, da sich das Trinkverhalten nach Transplantation negativ auf die Erfolgsaussichten der Transplantation auswirkt und daher von besonderem Interesse ist (Mellinger und Volk 2018, S. 175).

Eine hohe prognostische Präzision ist insbesondere deswegen zu fordern, da das Instrument dazu genutzt werden soll, einzelne Personen von einer potentiell lebenserhaltenden Ressource auszuschließen. Weder bezüglich der Prognose der Erfolgsaussicht einer Transplantation, sei es in Form des Patienten- oder des Transplantatüberlebens, noch des Trinkverhaltens nach Transplantation kann allerdings davon die Rede sein, dass die Validität der sechsmonatigen Karenzregel einer strengen wissenschaftlichen Prüfung unterworfen wurde (Al-Saeedi et al. 2018; Mathurin et al. 2011). Die fehlende Evidenz wird selbst von Verfechtern der Sechsmonatsregel offen zugestanden (Greif-Higer 2015). Gerade bei der Einschätzung des Trinkverhaltens nach Transplantation scheinen andere Faktoren, wie das familiäre und soziale Umfeld, psychische Begleiterkrankungen usw. eine weitaus bessere Prognose zu ermöglichen als eine starre Sechsmonatsregel (Im et al. 2018; Wu et al. 2018; Obed et al. 2018; Mathurin et al. 2011). Innerhalb der Suchtmedizin wird eine fünfjährige Abstinenz als einzige klinisch belastbare Karenzzeit für eine zuverlässige Vorhersage der weiteren Alkoholabstinenz von Patient*innen mit entsprechender Suchtproblematik gehandelt - eine Periode, die die meisten Patient*innen mit alkoholbedingtem Leberversagen nicht überleben würden (Testino et al. 2014, S. 14.645).

Die prognostische Validität wird zudem durch die Heterogenität der Daten und mangelnde Vergleichbarkeit der verfügbaren Studienergebnisse in Frage gestellt. Die Studien legen in ihren klinisch-empirischen Untersuchungen zum einen sehr unterschiedliche Definitionen dessen zu Grunde, was als Rückfall und damit als relevanter Endpunkt der Studie anzusehen ist. Zum anderen kommen in den Studien ganz unterschiedliche Messmethoden (z. B. unterschiedliche labordiagnostische Tests, Befragung der Patient*innen) zur Anwendung, die das Ausmaß an gemesse- 
nen Rückfällen wesentlich beeinflussen dürften (Mellinger und Volk 2018, S. 175; Testino et al. 2014, S. 14.648).

Auch die Einschätzung der Erfolgsaussichten und des Trinkverhaltens nach Transplantation lassen sich sowohl patienten- als auch allokationsorientiert ethisch begründen, wobei diesen beiden Begründungsstrategien jeweils unterschiedliche Interpretationen des Begriffs der Erfolgsaussichten zu Grunde zu legen sind. Eine patientenorientierte Einbeziehung der Erfolgsaussichten, d.h. eine solche, die an dem individuellen Wohl der Patient*innen orientiert ist, fokussiert darauf, für jede*n einzelne*n Patient*in die Therapieoptionen mit den jeweils besten Erfolgsaussichten zu bestimmen. Die Sechsmonatsregel kann aufgrund der fehlenden studienbasierten Evidenz nun aber gerade keinen zuverlässigen Vergleich der Erfolgsaussichten mit und ohne Transplantation leisten. Bereits veröffentlichte Studien legen vielmehr nahe, dass die Lebertransplantation für eine ganze Reihe von Patient*innen auch ohne Abwarten der sechsmonatigen Karenzzeit die erfolgversprechendere Therapieoption darstellt (Al-Saeedi et al. 2018; Batra und Wiesing 2018; Mellinger und Volk 2018, S. 175). Als patientenorientierte Einbeziehung der Erfolgsaussichten lässt sich die Karenzregel in der gegenwärtig in Deutschland angewandten Form demnach kaum rechtfertigen.

Ein prognostischer Test ist nur dann als medizinisch indiziert zu rechtfertigen, wenn dieser dazu geeignet ist, im Einzelfall gesichertes Wissen zu dem RisikoNutzen-Profil einzelner Therapieoptionen zu generieren (vgl. Wiesing 2017, S. 64). Dass die Richtlinien der BÄK dieser Forderung zumindest jenseits der Gruppe an Patient*innen mit alkoholbedingten Lebererkrankungen nachzukommen versuchen, zeigt sich an den diversen Ausnahmen, die unter Punkt III.6.2.2.2 von dem regulären, mittels laborchemischer Parameter errechneten MELD-Score (Model for Endstage Liver Disease) formuliert werden (Bundesärztekammer 2019). Der MELD-Score dient als prognostischer Score, der die Dringlichkeit der Patient*innen in Form der Drei-Monats-Mortalität abbilden soll, d.h. die Wahrscheinlichkeit, innerhalb der nächsten drei Monate ohne Transplantation zu versterben. Da der reguläre, gemessene MELD-Score (auch labMELD genannt) nach klinischer Einschätzung die Dringlichkeit bei bestimmten Lebererkrankungen nur unzureichend abbildet und also keine hinreichende Validität besitzt, wurden eine Reihe von Ausnahmen formuliert, bei denen eine gesonderte Festsetzung der Dringlichkeit mittels eines eigens zugewiesenen und in regelmäßigen zeitlichen Abständen erhöhten, sogenannten matchMELDs erfolgt (Bundesärztekammer 2019). Entsprechend ist für die Karenzregel zu fordern, dass hier eine stärker patientenorientierte, an der individuellen Erfolgsaussicht ausgerichtete Validität des Prognoseinstrumentes gewährleistet wird.

Eine allokationsorientierte Einbeziehung der Erfolgsaussichten stellt hingegen weniger auf die Identifikation der für die einzelnen Patient*innen besten Therapieformen $a b$, sondern auf die durchschnittlichen Überlebens- oder Organfunktionsraten des gesamten Kollektivs der Lebertransplantierten. Aus allokationsethischer Sicht steht die Frage im Vordergrund, wie stark die Erfolgsaussichten in die Allokationsentscheidungen miteinbezogen werden sollen und dürfen. Ein utilitaristisch motivierter Verteilungsmodus, der vornehmlich auf die Erfolgsaussichten einer Transplantation abstellt, riskiert in der Praxis all diejenigen Patient*innen von der Ressource auszuschließen, die als besonders bedürftig anzusehen sind (Bob- 
bert und Ganten 2013). An derartige Gerechtigkeitserwägungen schließt auch der Einwand an, dass die Sechsmonatsregel bisweilen höhere Anforderungen an die Patient*innen mit alkoholbedingten Lebererkrankungen stellt als an die anderen Patient*innengruppen (Marckmann 2015a). So ist es zwar erwiesen, dass ein Rückfall in alte Trinkgewohnheiten die Langzeitergebnisse der Transplantation negativ beeinflussen. Allerdings zeigen auch diese Patient*innen immer noch Ergebnisse, die vergleichbar mit den Langzeitergebnissen anderer Patient*innengruppen sind (Obed et al. 2018; Schmeding et al. 2011; Testino et al. 2014, S. 14.648). Dies ist auch einer der Gründe, weshalb die Karenzregel in Deutschland zum Teil als verfassungswidrig eingestuft wird (Dannecker und Streng-Baunemann 2014).

\section{Konklusion}

Aus den medizinischen Fachpublikationen und den Richtlinien zur Lebertransplantation lassen sich vier sprachlich vermittelte Interpretationsrahmen rekonstruieren, die eine jeweils unterschiedliche Wahrnehmung der sechsmonatigen Karenzzeit vermitteln. Diese sprachlich vermittelten Deutungsmuster werden jeweils als Argumentationsgrundlage für eine (vorübergehende) Einschränkung des Indikationsgebiets zur Lebertransplantation genutzt. In der medizinischen Praxis sind Einschränkungen des Indikationsgebiets gemeinhin in Bezug auf die mit einem Eingriff verbundenen Risiken und/oder Erfolgsaussichten zu rechtfertigen (Maio 2015, S. 76f.; Marckmann 2015b, S. 118 f.; Neitzke 2015, S. 84 f.; Wiesing 2017, S. 66, 72f.). Keiner der drei, in dem vorliegenden Beitrag näher betrachteten Interpretationsrahmen vermag es, eine hinreichende Rechtfertigung für eine derartige Einschränkung zu liefern.

In den ersten drei Interpretationsrahmen ist selbst wiederum die Frage der medizinischen Indiziertheit zu stellen. Die Karenzregel gerät hier vornehmlich als medizinisch-diagnostisches und prognostisches Instrument in den Blick. Der Einsatz eines diagnostischen oder prognostischen Werkzeuges in der medizinischen Praxis setzt voraus, dass dieses mit hinreichender Sicherheit den jeweiligen Zweck zu realisieren erlaubt und es keine alternativen Instrumente gibt, die hierzu besser geeignet sind. Diese Bedingungen sind gegenwärtig für keinen der drei Interpretationsrahmen hinreichend erfüllt. Insofern die medizinische Indikation zusammen mit dem Patientenwillen notwendige Voraussetzung für die ethische Zulässigkeit des Einsatzes einer therapeutischen, diagnostischen oder auch präventiven Intervention ist, lässt sich der Einsatz der sechsmonatigen Karenzregel zur (vorübergehenden) Einschränkung des Indikationsgebiets der Lebertransplantation bei alkoholbedingten Lebererkrankungen ethisch kaum rechtfertigen.

Die Richtlinien der BÄK zur Organtransplantation erfüllen eine doppelte Funktion. Zum einen sollen sie die Einschränkung des Indikationsgebiets für Organtransplantationen möglichst genau umreißen und rechtfertigen, zum anderen einheitliche Kriterien für die Verteilung der Organe unter den Patient*innen auf den Wartelisten formulieren. Die Prüfung der Indiziertheit einer Lebertransplantation geht der Zuteilung eines Spenderorgans logisch und zeitlich voraus. Sie ist im Prozess der Organallokation mit der Entscheidung über die Aufnahme auf die Warteliste gleichzusetzen. Eine starre Anwendung der sechsmonatigen Karenzregel lässt sich, wie 
gesehen, an dieser Stelle des Prozesses der Organallokation nicht rechtfertigen. Inwiefern das Vorliegen einer alkoholbedingten Lebererkrankung oder ein weiterhin bestehender Alkoholkonsum als Allokationskriterium bei der Verteilung der Spenderorgane unter den Patient*innen auf den Wartelisten angewendet werden kann, ist eine hiervon getrennte Frage, die auch nicht mehr die Frage der Eingrenzung des Indikationsgebietes oder der medizinischen Indiziertheit der Karenzregel betrifft. Hier schließen die bereits erwähnten, aber nicht näher diskutierten Überlegungen zur retrospektiven und prospektiven Verantwortung als Allokationskriterium in der Leberallokation an. Wie auch immer die mit dem vierten Interpretationsrahmen verbundenen ethischen Fragen zu beantworten sind, so deutet die bisherige Analyse dennoch bereits an, dass die prospektive Verantwortung für das Einhalten einer Karenz, wenn überhaupt, erst bei der Verteilung der Spenderlebern unter den Patient*innen auf der Warteliste als Allokationskriterium angewendet werden sollte (vgl. Veatch und Ross 2015, S. 315 f.).

Funding Open Access funding provided by Projekt DEAL.

Open Access Dieser Artikel wird unter der Creative Commons Namensnennung 4.0 International Lizenz veröffentlicht, welche die Nutzung, Vervielfältigung, Bearbeitung, Verbreitung und Wiedergabe in jeglichem Medium und Format erlaubt, sofern Sie den/die ursprünglichen Autor(en) und die Quelle ordnungsgemäß nennen, einen Link zur Creative Commons Lizenz beifügen und angeben, ob Änderungen vorgenommen wurden.

Die in diesem Artikel enthaltenen Bilder und sonstiges Drittmaterial unterliegen ebenfalls der genannten Creative Commons Lizenz, sofern sich aus der Abbildungslegende nichts anderes ergibt. Sofern das betreffende Material nicht unter der genannten Creative Commons Lizenz steht und die betreffende Handlung nicht nach gesetzlichen Vorschriften erlaubt ist, ist für die oben aufgeführten Weiterverwendungen des Materials die Einwilligung des jeweiligen Rechteinhabers einzuholen.

Weitere Details zur Lizenz entnehmen Sie bitte der Lizenzinformation auf http://creativecommons.org/ licenses/by/4.0/deed.de.

\section{Einhaltung ethischer Richtlinien}

Interessenkonflikt N. Primc gibt an, dass kein Interessenkonflikt besteht.

Ethische Standards Für diesen Beitrag wurden von der Autorin keine Studien an Menschen oder Tieren durchgeführt. Für die aufgeführten Studien gelten die jeweils dort angegebenen ethischen Richtlinien.

\section{Literatur}

Al-Saeedi M, Barout M, Probst P, Khajehl E, Weiss KH, Diener MK, Mehrabi A (2018) Meta-analysis of patient survival and rate of alcohol relapse in liver-transplanted patients for acute alcoholic hepatitis. Langenbecks Arch Surg 403:825-836. https://doi.org/10.1007/s00423-018-1720-z

Batra A, Wiesing U (2018) Zur ethischen und wissenschaftlichen Fragwürdigkeit der „Karenzklausel“ bei alkoholabhängigen Patienten auf der Warteliste zur Lebertransplantation. Sucht 64:7-9. https://doi. org/10.1024/0939-5911/a000519

Bird GLA, O'Grady JG, Harvey FAH, Calne RY, Williams R (1990) Liver transplantation in patients with alcoholic cirrhosis: selection criteria and rates of survival and relapse. BMJ 301:15-17

Bobbert M, Ganten TM (2013) Liver allocation: urgency of need or prospect of success? Ethical considerations. Clin Transplant 27(Suppl. 25):34-39. https://doi.org/10.1111/ctr.12154 
Bramstedt KA, Jabbour N (2006) When alcohol abstinence criteria create ethical dilemmas for the liver transplant team. J Med Ethics 32:263-265. https://doi.org/10.1136/jme.2005.012856thur

Bundesärztekammer (2019) Richtlinien zur Organtransplantation gem. § 16 TPG. Richtlinie gemäß $§ 16$ Abs. 1 S.1 Nrn. 2 u. 5 TPG für die Wartelistenführung und Organvermittlung zur Lebertransplantation. Dtsch Arztebl. https://doi.org/10.3238/arztebl.2019.rili_baek_OrgaWlOvLeberTx20190924

Cohen C, Benjamin M, Ethics and Social Impact Committee of the Transplant and Health Policy Center (1991) Alcoholics and liver transplantation. JAMA 265:1299-1301

Dannecker G, Streng-Baunemann AF (2014) Verschaffung des Wartelistenzugangs für Alkoholiker entgegen den Organallokations-Richtlinien der Bundesärztekammer - (versuchter) Totschlag? Neue Z Strafrecht 39:673-680

Donckier V, Lucidi V, Gustot T, Moreno C (2013) Ethical considerations regarding early liver transplantation in patients with severe alcoholic hepatitis not responding to medical therapy. J Hepatol 60:j866-j871

Entman RM (1993) Framing: toward clarification of a fractured paradigm. J Commun 43:51-58

Glannon W (2009) Responsibility and priority in liver transplantation. Camb Q Healthc Ethics 18:23-35. https://doi.org/10.1017/S0963180108090051

Greif-Higer G (2015) Alkoholabstinenz vor Lebertransplantation. Dtsch Arztebl 112:A2078

Ho D (2008) When good organs go to bad people. Bioethics 22:77-83. https://doi.org/10.1111/j.14678519.2007.00606.x

Im GY, Cameron AM, Lucey MR (2018) Liver transplantation for alcoholic hepatitis. J Hepatol 70:328-334. https://doi.org/10.1016/j.jhep.2018.11.007

Lee BP, Mehta N, Platt L, Gurakar A et al (2018) Outcomes of early liver transplantation for patients with severe alcoholic hepatitis. Gastroenterology 155:422-430. https://doi.org/10.1053/j.gastro.2018.04. 009

Maio G (2015) Die Indikation als Vertrauensgrundlage der Medizin. In: Dörries A, Lipp V (Hrsg) Medizinische Indikation. Ärztliche, ethische und rechtliche Perspektiven. Grundlagen und Praxis. Kohlhammer, Stuttgart, S 74-93

Marckmann G (2015a) Contra Alkoholabstinenz vor Lebertransplantation. Dtsch Arztebl 112:A2079

Marckmann G (2015b) Wirksamkeit und Nutzen als alternative Konzepte zur medizinischen Indikation. In: Dörries A, Lipp V (Hrsg) Medizinische Indikation. Ärztliche, ethische und rechtliche Perspektiven. Grundlagen und Praxis. Kohlhammer, Stuttgart, S 113-124

Marroni CA, de Medeiros Fleck A, Fernandes SA, Galand LH, Mucenic M, de Mattos Meine MH, Mariante-Neto G, de Mello Brandao AB (2018) Liver transplantation and alcoholic liver disease: history, controversies, and considerations. World J Gastroenterol 24:2785-2805. https://doi.org/10.3748/wjg. v24.i26.2785

Mathurin P, Moreno C, Samuel D, Dumortier J, Salleron J, Durand F et al (2011) Early liver transplantation for severe alcoholic hepatitis. N Engl J Med 365:1790-1800. https://doi.org/10.1056/ NEJMoa1 105703

Mellinger JL, Volk ML (2018) Transplantation for alcohol-related liver disease: Is it fair? Alcohol Alcohol 53:173-177. https://doi.org/10.1093/alcalc/agx105

Moss AH, Siegler M (1991) Should alcoholics compete equally for liver transplantation? JAMA 265:1295-1298

Neitzke (2015) Medizinische und ärztliche Indikation - zum Prozess der Indikationsstellung. In: Dörries A, Lipp V (Hrsg) Medizinische Indikation. Ärztliche, ethische und rechtliche Perspektiven. Grundlagen und Praxis. Kohlhammer, Stuttgart, S 83-93

Obed A, Bashir A, Stern S, Jarrad A (2018) Severe acute alcoholic hepatitis and liver transplant: a neverending mournful story. Clin Mol Hepatol 24:358-366. https://doi.org/10.3350/cmh.2018.0044

Schmeding M, Heidenhain C, Neuhaus R, Neuhaus P, Neumann UP (2011) Liver transplantation for alcohol-related cirrhosis: a single centre long-term clinical and histological follow-up. Dig Dis Sci 56:236-243. https://doi.org/10.1007/s10620-010-1281-7

Solga SF, Goldberg DS, Spacek LA, Forde KA (2018) Comment on B Lee et al outcomes of early liver transplantation for patients with severe alcoholic hepatitis. Gastroenterology 156:284-292. https:// doi.org/10.1053/j.gastro.2018.06.096

Testino G, Burra P, Bonino F, Piani F, Sumberaz A, Peressutti R, Castiglione AG, Patussi V, Fanucci T, Ancarani O, De Cerce G, Iannini AT, Greco G, Mosti A, Durante M, Babocci P, Quartini M, Mioni D, Aricò S, Baselice A, Leone S, Lozer F, Scafato E, Borro P (2014) Acute alcoholic hepatitis, end stage alcoholic liver disease and liver transplantation: An Italian position statement. World J Gastroenterol 20:14642-14651. https://doi.org/10.3748/wjg.v20.i40.14642 
Thornton V (2009) Who gets the liver transplant? The use of responsibility as the tie breaker. J Med Ethics 35:739-742. https://doi.org/10.1136/jme.2009.029967

Veatch RM, Ross LF (2015) Transplantation ethics. Georgetown University Press, Washington DC

Wiesing U (2017) Indikation. Theoretische Grundlagen und Konsequenzen für die ärztliche Praxis. Kohlhammer, Stuttgart

Wu T, Morgan TR, Klein AS, Volk ML, Saab S, Sundaram V (2018) Controversies in early liver transplantation for severe alcoholic hepatitis. Ann Hepatol 17:759-768. https://doi.org/10.5604/01.3001.0012. 3134

Zambrano A (2016) Why alcoholics ought to compete equally for liver transplants. Bioethics 30:689-697 